\title{
Les regards des extrêmes droites italiennes sur le combat pour l'« Algérie française »: circulations et transferts (années 1960)
}

Lo sguardo delle estreme destre italiane sulla lotta per l'Algeria francese:

circolazione e trasferimenti negli anni Sessanta

The View of the Italian Extreme Rights on the Fight for "French Algeria":

Circulation and Transfers during the Sixties

\section{Pauline Picco}

\section{OpenEdition} Journals

\section{Édition électronique}

URL : http://journals.openedition.org/cei/2909

DOI : 10.4000/cei.2909

ISSN : 2260-779X

\section{Éditeur}

UGA Éditions/Université Grenoble Alpes

\section{Édition imprimée}

Date de publication : 20 avril 2016

Pagination : 127-140

ISBN : 978-2-84310-323-0

ISSN : 1770-9571

\section{Référence électronique}

Pauline Picco, « Les regards des extrêmes droites italiennes sur le combat pour I' « Algérie française »: circulations et transferts (années 1960) », Cahiers d'études italiennes [En ligne], 22 | 2016, mis en ligne le 01 janvier 2017, consulté le 26 mars 2021. URL : http://journals.openedition.org/cei/2909; DOI : https://doi.org/10.4000/cei.2909 


\title{
LES REGARDS DES EXTRÊMES DROITES ITALIENNES SUR LE COMBAT POUR L'«ALGÉRIE FRANÇAISE » : CIRCULATIONS ET TRANSFERTS (ANNÉES 1960)
}

\author{
Pauline Picco \\ Université Paris-Sorbonne, Labex EHNE-SIRICE
}

Dès le début des années 1960, les relations franco-italiennes ont été troublées par la présence dans la péninsule d'activistes se réclamant du combat pour l'Algérie française puis de l'Organisation armée secrète (OAS). Créée en février 196I, cette dernière s'attache, par des actions de propagande et par un activisme terroriste, à combattre le Front de libération nationale (FLN) algérien. Dès 196I, alors que l'organisation est farouchement combattue par celui-ci et par le gouvernement français, les activistes se mettent à la recherche de nouveaux territoires européens sur lesquels appuyer leur combat. Si l'Espagne et son régime franquiste présentent des facilités d'accueil pour ces activistes, une quinzaine de militants pour l'Algérie française voient dans la péninsule italienne une terre disposée à les accueillir. Ces militants se réclamant de l'OAS et, réfugiés en Italie, ont en effet bénéficié de la bienveillance de certaines branches des services de renseignements ${ }^{\mathrm{I}}$ et du soutien de membres de la Curie romaine et de la Démocratie chrétienne (DC) proches du courant tambronien ${ }^{2}$, alors même que l'Italie est le lieu d'élaboration d'un attentat contre de Gaulle en août 1964 ${ }^{3}$. Le gouvernement italien, loin d'adopter une politique ferme à l'égard de l'OAS semble avoir tergiversé, oscillant entre un attentisme bienveillant et une politique d'expulsions ciblées. Parallèlement, les menaces — véritables

I. Nous désignons ici la Divisione Affari riservati du ministère de l'Intérieur et surtout le SIFAR (Servizio informazioni forze armate).

2. Né en I90I, le démocrate-chrétien Fernando Tambroni est plusieurs fois ministre de l'Intérieur avant de présider, en mai 1960, un gouvernement qui bénéficie des voix du MSI. Il autorise, en juillet 1960, la tenue du congrès national du MSI à Gênes, ville médaille d'or de la Résistance, ce qui provoque de grandes manifestations, violemment réprimées par la police.

3. Il s'agit de l'attentat du mont Faron perpétré contre de Gaulle le is août I964. 
ou fantasmées - qui pesèrent sur la vie d'Enrico Mattei, président de l'ENI et figure majeure de l'économie et de l'État italien, contribuent, à gauche, au lancement d'une campagne de presse qui dénonce le péril qu'une alliance possible entre le Movimento sociale italiano (MSI), principal parti de l'extrême droite italienne d'après-guerre, et l'OAS, ferait peser sur la démocratie italienne. Ce sont en effet les milieux de la droite et de l'extrême droite italiennes qui apportent leur soutien verbal, symbolique ou effectif, aux activistes en déroute. Ces appuis, s'ils émanent de diverses structures, partis et groupes politiques unis par un même anticommunisme viscéral, ont en commun un ancrage qui se situe indéniablement à droite de l'échiquier politique. Certains courants de la Démocratie chrétienne, plusieurs membres de la Curie romaine, une partie du MSI et des groupes extra-parlementaires ont favorisé le séjour des activistes français dans la Péninsule.

C'est sur ces groupes d'extrême droite ${ }^{4}$ et les regards qu'ils portent sur le combat pour l'Algérie française que nous porterons notre attention. Il s'agit en effet de montrer comment ils perçoivent et se réapproprient ce combat "Algérie française", durant les années 1960, bien après l'accession à l'indépendance de l'Algérie en juillet 1962. Au-delà de ce décalage qui met en lumière l'importance des décalages chronologiques et des discordances des temps en matière de transferts politiques et culturels, comment la présence d'activistes OAS marque-t-elle les extrêmes droites italiennes? Comment contribue-t-elle à poser les jalons d'une culture politique commune à celles-ci et à leurs homologues françaises en favorisant les phénomènes de transferts et de circulations de part et d'autre des Alpes? À travers cette étude, il s'agira de montrer comment l'exil des militants OAS affecte à plusieurs niveaux la vie politique et l'opinion publique italiennes.

Dès 1960, l'extrême droite extraparlementaire italienne manifeste sa solidarité et son soutien à l'égard du combat pour l'Algérie française tandis que le MSI est divisé sur le soutien à apporter aux militants OAS présents en Italie. Ensuite, le «mythe OAS» s'impose, après le printemps 1962 et

4. Notre étude s'appuie sur les périodiques produits par le parti et les groupes suivants : Movimento sociale italiano (MSI), Centro studi Ordine Nuovo (ON), Formazioni nazionali Giovanili (FNG), L'Italiano. Nous avons dépouillé environ 45 numéros du périodique Ordine Nuovo de 1955 à 1972 (environ trois quarts des numéros parus), la collection complète de L'Italiano de 1959 à 1984 (II2 numéros), le quotidien du MSI Il secolo d'Italia de janvier 196I à décembre 1968 puis entre juillet et décembre 1969. Nous avons également procédé au dépouillement systématique de la revue française Europe-Action (janvier 1963-novembre 1966, 46 numéros) qui compte parmi ses rédacteurs d'anciens militants de l'OAS et entretient d'étroits contacts avec ceux d'Ordine Nuovo. Enfin, nous avons complété notre corpus par les archives du cabinet du ministère de l'Intérieur italien et celles de la Casa della Memoria de Brescia. 
durant toute la décennie, comme une matrice du combat nationaliste dans la Péninsule.

\section{Le soutien précoce de l'extrême droite extraparlementaire italienne au combat pour l'Algérie française}

Tous les soutiens italiens à l'OAS ont gravité, de près ou de loin, autour des groupes les plus radicaux de l'extrême droite italienne, et notamment le Centro studi Ordine Nuovo ${ }^{5}$, créé en 1954 et dirigé par Pino Rauti ${ }^{6}$. Dès novembre 1960, sa revue, Ordine Nuovo exalte le combat pour l'Algérie française dans ses colonnes et porte un regard plein d'espoir sur le contexte politique en métropole qualifié de "pré-révolutionnaire» 7 .

En janvier 196I, les militants des sections locales du Centro studi Ordine Nuovo manifestent leur soutien aux activistes français en publiant des tracts au contenu explicite qui fustigent cette " "révolution de couleur", [c]es organisations de "jeunesse" social-communistes, unies aux sans-patries et aux homosexuels de l'internationale radical-marxiste» qui s'opposerait à «l'opinion publique française rassemblée autour de LAGAILLARDE (sic), de la Jeune nation et des Ultras ${ }^{8}$ ». Ordine Nuovo définit ici une figurerepoussoir du "paria" ", caractéristique d'une rhétorique raciste et antisémite appartenant au patrimoine idéologique de l'extrême droite : celle d'un adversaire subversif, homosexuel, dévirilisé et apatride, dont la «jeunesse» est remise en cause puisque celle-ci est réputée porteuse de valeurs positives. Ordine Nuovo contribue également à faire naître le mythe des "paras", définis comme les " "centurions" d'un principe ou d'une idée à venir ${ }^{\mathrm{IO}}$ ».

5. Ordine Nuovo constitue la principale alternative extraparlementaire à la politique menée par le Movimento sociale italiano, jugé trop modérée. Fortement influencé par Julius Evola, Ordine Nuovo (ON) se réclame de la République sociale italienne (RSI) et incarne une tendance culturelle et idéologique singulière dans le monde de l'extrême droite italienne.

6. Né en 1926, Rauti effectue son service militaire comme sous-lieutenant de la Garde nationale républicaine de la République sociale italienne. Il adhère au MSI en 1946 et acquiert rapidement un rôle de premier plan au sein de ses organisations de jeunesse. Proche de Julius Evola, il fonde en 1954 le groupe Ordine Nuovo qui fait scission du MSI à partir de 1956 et mène une existence autonome jusqu’en 1969. Il meurt en 2012.

7. G. Fergola, «Possibilità e prospettive per la 'controrivoluzione'», Ordine Nuovo, vol. IV, $\mathrm{n}^{\circ} 4-5$, novembre I960, p. 66-80.

8. Archivio centrale dello Stato (ACS), Ministero dell'Interno (MI), Gabinetto, 1967-1970, busta 25, fasc. "Movimeno politico Ordine Nuovo varie», tract annexé au rapport d'information spécial du groupe de carabiniers de Messine, i9 janvier 196r.

9. G. L. Mosse, L'image de l'homme. L'invention de la virilité moderne, Paris, Pocket, I999 (1996), p. 69 et suivantes.

IO. S. Mangiante, «La destra in Francia non rispetta gli idoli», Ordine Nuovo, vol. VII, février-mars I96I, p. 30-34. 
Les Formazioni nazionali giovanili (FNG) constituent le deuxième pôle de la droite extraparlementaire, étroitement lié au MSI et engagé dans le soutien à l'OAS II. En partie financées par Luigi Gedda et Vanni Teodorani ${ }^{\mathrm{I} 2}$, elles furent, selon le journaliste d'extrême droite Fabrice Laroche $^{13}$, chargées de "soutenir la politique "Algérie française", éventuellement d'aider quelques clandestins, ceci toujours à titre presque exclusivement personnel ${ }^{\mathrm{I} 4}$ ». Ces propos sont partiellement confirmés par une note des services civils de renseignements datée du 8 juin 196I qui indique que les FNG diffusent des imprimés dédiés à la question de l'Algérie française ${ }^{15}$. En août 196I, certains membres de l'organisation se seraient déplacés en Europe pour rencontrer les dirigeants d'autres associations. Après Amsterdam, Hambourg, Malmö, ils se rendent à Nice, où «ils ont rencontré de jeunes membres d'extrême droite appartenant à l'OAS et un jeune Français qui fait la navette en permanence entre la France, l'Italie et l'Espagne pour des raisons politiques, qui doivent être liées à l'action que les droites françaises effectuent sur le territoire métropolitain et en Algérie $^{16}{ }^{\prime}$. Ces contacts sont en outre certainement favorisés par Guido Giannettini qui fait alors office d'intermédiaire entre extrême droite italienne et groupes OAS en Algérie ${ }^{17}$. Le combat OAS semble avoir fait naître à la politique internationale cet homme de réseau, qui demeure, durant deux décennies, un personnage pivot, relais entre les extrêmes droites européennes et les services de renseignement ${ }^{18}$.

Le soutien apporté n'est pas uniquement rhétorique, il est également, pour partie concret et trouve son expression dans l'action politique. Une

II. En juillet 1962, l'organisation recense une petite centaine d'adhérents. Casa della Memoria di Brescia, Dossier de procédure pénale n ${ }^{\circ}$ II/97 mod. 2I, ADCPP, fasc. «Formazioni nazionali giovanili», lettre de la Préfecture de police de Rome aux préfectures et au ministère de l'Intérieur, DGPS, AA. RR., AA. GG., 3 juillet 1962.

I2. Les deux hommes sont liés aux réseaux démocrates-chrétiens proches de Fernando Tambroni. Gedda est le fondateur des Comitati civici de la DC. Teodorani est un député MSI, marié à la nièce de Mussolini, ancien de la République sociale italienne (RSI), il est directeur du périodique Rivista romana.

13. Fabrice Laroche est le pseudonyme utilisé par Alain de Benoist dans les années 1960. Né le II décembre 1943, il écrit ses premiers articles dans Lectures françaises d'Henry Coston. En 1963, il participe à la création d'Europe-Action et en devient l'un des principaux collaborateurs.

I4. F. Laroche, "Le MSI, des origines à nos jours", Europe-Action, nº II, novembre 1963.

I5. Casa della Memoria, Dossier de procédure pénale nº 9I/97 mod. 2I, ADCPP, fasc. «Formazioni nazionali giovanili ", lettre réservée de la Préfecture de police de Rome au ministère de l’Intérieur, DGPS, div. AA. GG. et AA. RR., 8 juin 196I (G-a-23).

I6. Casa della Memoria, Dossier de procedure pénale nº 9I/97 mod. 2I, ADCPP, fasc. «Formazioni nazionali giovanili", 3I août I96I (G-a-23).

17. Casa della Memoria, Rapport d'expertise n 6 d'Aldo Giannuli, Annexe ro5, ADCPP, fasc. "Oas», Note du 29 décembre i961.

I8. Né en 1931, Giannettini est journaliste. Militant du MSI à la fin des années I950, il se rapproche ensuite des groupes extraparlementaires radicaux. Au milieu des années 1960, il est proche des milieux militaires et des services de renseignements. Il devient officiellement collaborateur du SID (Servizio informazioni difesa) en I967. Il meurt en 2003. 
partie de la jeunesse italienne d'extrême droite fait, grâce à la présence d'activistes se réclamant de l'organisation secrète et à leur combat, sa première expérience de solidarité militante. Une note de l'informateur Aristo $^{19}$ datée de novembre 1963, portant sur les trafics d'armes pratiqués par Ordine Nuovo au profit du Portugal "pour soutenir l'effort de guerre en Angola", fait référence aux services qu'aurait rendus le groupe italien à l'OAS. Un ancien lieutenant des "paras» français, alors installé à Lisbonne aurait eu

[...] l'occasion d'expérimenter personnellement les compétences de Rauti et de ses amis. En effet, cet ancien militaire français, déserteur durant la révolte militaire contre de Gaulle, a été blessé par la police et, grâce aux liens avec Ordine Nuovo, on lui a fait traverser la frontière dans le coffre d'une voiture avec sa fiancée, et on l'a soigné dans une clinique privée. Il s'est avéré qu'Ordine Nuovo a une certaine expérience dans ce genre d'entreprise et, si l'on s'en tient à ce que Rauti a admis, on a fait à plusieurs reprises passer la frontière à des chargements d'armes destinés à des membres de l'OAS, grâce à la collaboration d'un agent des douanes, ancien membre des FAR ${ }^{20}$ qui, il y a quelques temps, exerçait à la frontière franco-italienne ${ }^{21}$.

Rauti va jusqu'à proposer l'envoi en Algérie d'une quarantaine de militants italiens sans que l'OAS ne donne suite.

Mais si l'extrême droite extraparlementaire porte un regard bienveillant et admiratif sur le combat des partisans de l'Algérie française et assume parfaitement le soutien apporté à l'OAS, ce n'est pas le cas du Movimento sociale italiano.

\section{MSI et OAS : les divisions du parti}

La position du Movimento sociale italiano (MSI), principal parti italien d'extrême droite, est loin d'être univoque et le soutien apporté aux activistes de l'OAS y suscite débats et divisions. Il fait l'objet de débats houleux. Les dirigeants du MSI peinent à saisir les tenants et les aboutissants d'une situation complexe, et ils ne semblent pas, au moins jusqu'au «putsch» d'Alger d'avril I96ı, donner de directives claires à leurs militants.

19. L'informateur Aristo, pseudonyme d'Armando Mortilla, appartient aux instances dirigeantes du Centro studi Ordine Nuovo. Il est considéré comme très fiable par les services de renseignements.

20. Le sigle désigne les Fasci d'azione rivoluzionaria, organisation terroriste qui commit des attentats contre des institutions de la République italienne au début des années I950. Un certain nombre de dirigeants du Centro studi Ordine Nuovo - Clemente Graziani, Pino Rauti, Paolo Andriani — sont issus des FAR.

2I. Casa della Memoria, Rapport d'expertise d'Aldo Giannuli commandité le I2 mars 1997, Annexe 92, APPMi, ADCPP, fasc. "Rauti Pino", note de l'informateur Aristo, 25 novembre 1963. 
Dans le journal du parti, Il Secolo d'Italia ${ }^{22}$, ils soutiennent successivement de Gaulle puis l'OAS. Ces atermoiements reflètent en réalité l'opposition des tendances qui traversent le parti.

Arturo Michelini, son secrétaire général, défend une stratégie légaliste et d' «insertion dans le système ${ }^{23}$ ", et il ne peut donc risquer de voir le parti compromis par les actions de l'organisation "ultra». Son démenti public et télévisé d'octobre 196r quant à l'existence de relations entre l'OAS et le MSI $^{24}$ vient confirmer cette hypothèse.

Au sein des instances dirigeantes et en opposition à Michelini, la tendance portée par Pino Romualdi ${ }^{25}$, l'un des fondateurs du parti, soutient ouvertement le combat de l'OAS. L'homme est auréolé du prestige de celui qui a participé à la guerre de conquête éthiopienne ${ }^{26}$ et à l'expérience fondatrice de la République de Salò. Il fait du périodique qu'il dirige, L'Italiano, le principal porte-voix en Italie du combat pour l'Algérie française, avant de défendre ouvertement l'action de l'OAS.

Dès février 1960, à la suite des événements de la semaine des barricades, sa revue consacre sa couverture à "la colère d'Alger» et souligne l'importance du combat pour l'Algérie française dans l'histoire de l'Occident. La "révolte d'Algérie» est en effet définie comme "la plus belle page de vie et de jeunesse écrite et vécue ces dernières années, au milieu de cet Occident pourri par la démocratie et par la vieillesse obscène ${ }^{27}$ ». L'auteur reprend ici une thématique classique caractéristique du nationalisme d'avant la Première Guerre mondiale, récupérée en son temps par le fascisme mussolinien, exaltant une jeunesse métaphorique opposée à la démocratie associée à la vieillesse. C’est donc la vertu régénératrice et révolutionnaire ${ }^{28} \mathrm{de}$ cette jeunesse qui est glorifiée.

Larticle critique fermement la politique d'autodétermination du général de Gaulle, synonyme de la perte de l'Algérie, bastion de l'Occident. C'est donc au nom d'un combat occidentaliste, européiste et anticommu-

22. Il Secolo d'Italia, quotidien créé en 1952 pour défendre les idées du MSI dans la perspective de l'échéance électorale de 1953, ne devient le quotidien officiel du MSI qu’en 1963. En 196I-I962, il est dirigé par un triumvirat composé de Franz Maria D’Asaro, Filippo Anfuso et Giorgio Almirante.

23. Il s’agit de la ligne politique adoptée par Arturo Michelini, secrétaire du MSI.

24. L'intégralité de l'entretien télévisé est rapportée par Il Secolo d'Italia, jeudi 5 octobre I96I, p. I et 8.

25. Il convient de préciser que si les deux hommes s'opposent sur le soutien à l'OAS, c'est notamment grâce au soutien de Pino Romualdi que Michelini s'impose à la tête du parti, en opposition, notamment, avec le courant Rinnovamento de Giorgio Almirante.

26. Nous faisons ici référence à la guerre menée par l'Italie pour conquérir l'Éthiopie, d'octobre 1935 à mai 1936.

27. “Una lettera dell'Italiano ", L'Italiano, vol. II, n 2, février I96o, p. 2.

28. L. Bantigny, «Les jeunes, sujets et enjeux politiques (France, $\mathrm{xx}^{\mathrm{e}}$ siècle)", Histoire@politique, $\mathrm{n}^{\circ} \mathrm{I}, 2008$, p. I. 
niste que Romualdi et sa revue s'engagent aux côtés des insurgés. Quelques mois plus tard, en juillet-août 1960, L'Italiano fait état de la situation en Algérie en insistant sur le fait que «Alger, Oran, Constantine, Philippeville sont des villes européennes, construites par des Européens et habitées dans leur majorité par des Européens ${ }^{29} »$. L'expérience coloniale italienne et sa mémoire sont invoquées pour mettre en parallèle la valorisation du territoire algérien par les Français et celle du territoire libyen par les Italiens. En vertu de cet exemple, l'auteur soutient l'initiative des Européens d'Algérie à refuser l'abandon du territoire algérien et encourage la droite française à poursuivre le combat, même face à de Gaulle ${ }^{30}$. Dans ce contexte troublé, l'Algérie et l'Union sud-africaine sont considérées comme les derniers bastions de l'Europe et de la race blanche en Afrique. Le discours occidentaliste se double d'une composante raciste, justifiée par l'affirmation d'une prétendue "mission civilisatrice» européenne en Afrique et la supposée appartenance "aux blancs, et seulement aux blancs", des "réalisations effectuées dans les deux territoires ». Utilisant une rhétorique raciste et colonialiste, l'auteur ajoute: «Les populations indigènes, trompées par la vénéneuse propagande soviétique, seraient restées à l'état primitif sans l'aide des Européens.» C'est donc au nom d'un farouche anticommunisme et de la défense d'une race blanche et de l'Occident, qu'à leurs yeux, «les dernières tranchées de l'Europe» doivent être défendues ${ }^{31}$.

Entre novembre 196I et juin-juillet 1963, Pino Romualdi, sans doute rappelé à l'ordre par Michelini, met en sourdine le soutien à l'OAS dans L'Italiano. Toutefois, il ne s'abstient pas d'interpeller le gouvernement à la Chambre des députés, le 5 mars 1962, jour de l'expulsion de Philippe de Massey, proche de Jo Ortiz et activiste de l'OAS ${ }^{32}$, dans une question adressée aux ministres de l'Intérieur et des Affaires étrangères, en réponse à une interrogation communiste ${ }^{33}$ sur le problème de l'OAS en Italie ${ }^{34}$. Il donne ainsi une résonance nationale à son soutien.

29. «Algeria algerina», L'Italiano, vol. II, no 7-8, juillet-août I960, p. I4-I5.

30. Ibid., p. I5.

3I. Ibid.

32. Né en 1929, Philippe de Massey est formé politiquement au sein de l'Action française. Engagé volontaire, il accède au grade de lieutenant et il combat pendant six mois en Algérie durant l'année 1956. Il adhère, en I959, à l'Association des combattants de l'Union française (ACUF) d'Yves Gignac. Recherché par les autorités françaises, il est exfiltré en Suisse où il est employé par une société de vente d'armes. Soupçonné d'être impliqué dans l'assassinat du maire d'Évian, il s'installe en Italie où il effectue de fréquents déplacements entre San Remo, Rome et Catane.

33. Camera dei deputati, III legislatura, discussioni, seduta del 5 marzo 1962, interrogazione dei deputati Alicata, Pajetta, Giuliano, Seroni, Lajolo, Natta, p. 27752.

34. Camera dei deputati, III legislatura, discussioni, seduta del 5 marzo 1962, interrogazione del deputato Romualdi, 5 mars I962, p. 27752. 
Le soutien verbal à l'OAS de Romualdi et des organes de presse qu'il dirige est complété par l'appui des organisations de jeunesse du MSI, sur lesquelles le dirigeant missin exerce une influence importante. Le fait générationnel apparaît fondamental dans le soutien du MSI aux activistes OAS. En effet, ce sont essentiellement ces organisations de jeunesse - le Fronte universitario di azione nazionale (FUAN) dirigé par Franco Petronio ${ }^{35}$, l'ASAN Giovane Italia ${ }^{36}$ dirigée par Massimo Anderson et le Raggruppamento giovanile studenti e lavoratori ${ }^{37}$ sous la direction de Giulio Caradonna ${ }^{38}$ - qui s'illustrent par leur appui à la lutte des partisans de l'Algérie française.

Les dirigeants des organisations de jeunesse ne sont pas les seuls à entretenir des relations suivies avec les activistes français : l'informateur Mauro $^{39}$ fournit ainsi, en mars 1962, une liste plausible de seize responsables politiques du MSI "qui ont eu des contacts avec les émissaires de l'OAS française» : y figurent les noms de Filippo Anfuso, Giorgio Almirante, Arturo Michelini, Nettuno — dit Pino - Romualdi, Giulio Caradonna affublé de l'adjectif « dangereux ${ }^{40}$ ».

En réalité, le soutien à l'OAS révèle les fractures politiques qui lacèrent le MSI tandis qu'une partie de ses jeunes militants saisit l'occasion pour s'opposer «au courant modéré qui voudrait imposer au parti une politique légaliste ${ }^{4 \mathrm{I}}$. Symptôme de la reprise en main opérée par le parti, les plus fervents soutiens à la cause OAS sont contraints de nier leur soutien effectif aux activistes en territoire italien : lors d'une réunion locale du FUAN en mars 1962, Franco Petronio se voit dans l'obligation de récuser les accusations portées contre cette organisation soupçonnée d'entretenir des relations avec l'OAS. À l'approche de la signature des accords d'Évian, il est fort probable que Petronio ait reçu des consignes de retenue. Toutefois, il semble que ces dernières ne soient que très partiellement respectées :

35. Le FUAN est la fédération universitaire du MSI. Franco Petronio, qui la dirige, est né en I93i à Trieste. 36. Associazione studentesca di azione nazionale Giovane Italia. Créé en I954, ce mouvement est l'organisation lycéenne du parti. L'empreinte d'Evola y est manifeste. Son texte fondateur est la "Carta della Gioventù " (charte de la jeunesse) écrite par le philosophe en I95I.

37. L'organisation est créée en 1949.

38. Le député Giulio Caradonna (1927-2009) est en outre le responsable national du secteur jeunesse du MSI. Sur son soutien à la cause de l'Algérie française, G. S. Rossi, "L'influenza della guerra d'Algeria sull'estrema destra italiana", dans A. Ventrone (éd.), I dannati della rivoluzione. Violenza politica e storia d'Italia negli anni Sessanta e Settanta, Macerata, EUM, p. 30.

39. L'informateur Mauro est vraisemblablement lié de manière étroite aux milieux MSI du nord de l'Italie. Les informations qu'il communique au chef de la police de Rome sont, dans l'ensemble, plutôt fiables.

40. Casa della Memoria, Rapport d'expertise d'Aldo Giannuli du I2 mars 1997, Annexe 23, MI, Archivio della polizia di prevenzione (APP-Mi), fasc. "Oas», Lettre «raccomandata espresso — réservée personnelle» de l'informateur Mauro au vice-chef de la police sur les rapports entre OAS et MSI, is mars 1962.

4I. Ibid. 
Fabio de Felice, député MSI proche d'Evola, ex-dirigeant de la Giovane Italia, se serait rendu en France au début du mois d'avril 1962, au mépris des directives de la direction pour y rencontrer le capitaine Sergent, "un des plus importants dirigeants des courants extrémistes de l'OAS, celle des "plastiquer" $(\text { sic })^{42} »$.

\section{Le «mythe OAS », matrice du combat nationaliste en Italie : quand l'Algérie française devient l'Algérie europénne}

Dès lors, et jusqu’au début des années 1970, l'organisation secrète constitue en Italie une matrice du combat nationaliste tandis que se constitue un «mythe OAS» fondamental au sein du panthéon militant et mémoriel de l'extrême droite italienne. Les Italiens érigent ainsi les "paras» et l'OAS en mythes matriciels et contribuent à favoriser, de part et d'autre des Alpes, des circulations, des transferts idéologiques et culturels qui nourrissent des constructions identitaires et des mémoires communes aux extrêmes droites. Une partie de l'extrême droite italienne fit sien le combat Algérie française, par un processus d'appropriation essentiellement fondé sur un déplacement sémantique.

En avril 1962, prenant acte de la signature des accords d'Évian, la section de Catanzaro d'Ordine Nuovo diffuse un tract dont le message est le suivant : "[...] Jeunes, souvenez-vous que le sang français est sang européen! En Algérie, on combat et on meurt pour l'Europe à laquelle vous appartenez [...]. L'Algérie doit être restituée à l'Europe.» L'Algérie française se transforme dès lors, dans le discours d'une partie de l'extrême droite italienne, en Algérie européenne et, dans l'esprit des activistes, l'Italie est redéfinie de manière rhétorique comme une puissance sinon coloniale, du moins occidentale. Il faut relever ici le décalage entre la chronologie de l'accession à l'indépendance de l'Algérie qui signe en partie la fin du combat OAS et la réappropriation par les Italiens du combat, érigé comme mythe mobilisateur à partir du printemps 1962. Le "mythe OAS" contribue ainsi à la formation d'un discours franco-italien de soutien à la lutte des activistes, qui semble avoir dépassé la rivalité coloniale francoitalienne, point d'achoppement entre les deux pays jusqu'à l'indépendance de la Tunisie en $1956^{43}$. Toutefois, un dépouillement systématique

42. Casa della Memoria, Dossier de procédure pénale n 9I/97 mod. 2I, ADCPP, fasc. "OI/2 Francia Oas", Rome, Note du 3 avril 1962 (G-a-52). Les erreurs d'orthographe et de grammaire sont dans le texte.

43. Cf. M. Tomassetti, Séquestre et liquidation des biens italiens en Tunisie (I940-1954) : derniers enjeux de la présence française, thèse de doctorat (dir. R. Ilbert), Aix-Marseille I, 2003. 
des périodiques d'extrême droite impliqués dans le soutien OAS et le témoignage des anciens militants français amènent à nuancer cet "oubli», relatif, d'une rivalité séculaire ${ }^{44}$. L'accusation porte, il est vrai, davantage sur la Grande-Bretagne, jugée responsable de l'expulsion de l'Italie de la Libye, que sur une France relativement épargnée par les critiques ${ }^{45}$.

Au-delà de cette rivalité, Ordine Nuovo manifeste régulièrement, de I962 à 1965 son soutien aux Français d'Algérie puis à ceux qui se réclament de ce combat. Cette bataille, pour défendre l'Europe et la civilisation est considérée comme un élément de la défense d'une "présence blanche en Afrique ${ }^{46} »$. Manifestant sa solidarité à l'égard des Européens d'Afrique, Pino Rauti écrit :

Nous avons fait «nôtre» la lutte héroïque et désespérée des Français d'Algérie, la tragédie des Belges massacrés au Congo, le drame des Portugais demeurés seuls à défendre en armes des "valeurs» qui sont celles de tout l'Occident civil et des Sud-Africains, dernier bastion assiégé par l'incompréhension du monde entier ${ }^{47}$.

Selon lui, la présence européenne en Afrique ne pouvait en aucun cas avoir eu un quelconque effet néfaste sur le continent, car les Européens n'y auraient "rien "volé" et [l'auraient] encore moins exploité $4^{8}$ ».

Le combat activiste en Algérie est en outre défini par Ordine Nuovo comme la «tragédie de l'honneur et de la fidélité à un drapeau ${ }^{49}$ ». Dans le conflit algérien, la notion d'honneur associée à celle du «respect de la parole donnée ${ }^{50}$ » est constamment invoquée par les activistes OAS, comme justification de leur combat. En réalité, comme l'écrit Eugen Weber, «en Algérie et concernant l'Algérie, toutes les parties font appel à l'honneur ${ }^{\mathrm{SI}}$ » et la tentative de réappropriation de ce concept est un enjeu d'autant plus important pour les extrêmes droites française et italienne que, dans les deux pays, la «Résistance fut identifiée à la défense de l'honneur : honneur national $[. .$.$] mais aussi honneur personnel { }^{52} »$. L'enjeu est donc de taille : il s'agit pour les militants de se réapproprier, face à certains héros

44. Nous faisons ici mention du dépouillement systématique de L'Italiano, du Secolo d'Italia et de Il Borghese pour les périodes concernées.

45. "La verità sulla rivolta di Algeri», numéro spécial de L'Italiano, vol. II, nº II, novembre I960; C. Di Risio, "Il Grande Maghreb», L'Italiano, vol. III, n 3, mars I96r.

46. P. Rauti, «Un Impero per l'Europa», Ordine Nuovo, vol. X, nº I-2, janvier-février 1964, p. I-7.

47. Ibid., p. 5 .

48. Ibid.

49. N. Mollicone, «La tragedia degli harkis», Ordine Nuovo, vol. IX, nº I, février 1963, p. 48-52.

50. G. Maceratini, "Vent’anni di “ingiustizia” ", Ordine Nuovo, vol. X, nº I-2, janvier-février 1964, p. I4-22. Sur ce point, voir : O. Dard, "L'armée française face à l'organisation armée secrète (OAS) », dans O. Forcade, É. Duhamel et P. Vial (éd.), Militaires en République 1870-1962, Paris, Publications de la Sorbonne, 1999, p. 690.

5I. E. Weber, "Vie et mort de l'honneur", Le Débat, nº 92, novembre-décembre 1996, p. I70.

52. Ibid., p. I69. 
de la Résistance honnies3 (essentiellement les communistes), une valeur qui appartient historiquement et fondamentalement à leur patrimoine identitaire. Le contexte historique, marqué en France comme en Italie par une série d'humiliations internationales et de camouflets, ne peut que renforcer la nécessité impérieuse de ce recours exclusif à l'honneur. Le couple honneur/déshonneur, auquel s'ajoute celui qui oppose la traitrise à la fidélité, structure donc fondamentalement l'imaginaire politique des activistes.

Avec ces derniers et les "paras", ce sont les harkis qui sont l'objet de toute la sollicitude et de la révolte des extrémistes italiens durant l'année 1963. Dès le mois de février, Ordine Nuovo, sous la plume de l'un de ses dirigeants, Nazzareno Mollicone ${ }^{54}$, dénonce la "vraie tragédie», celle des «fidèles musulmans, [des] harkis qui ont vu dans le Français et dans l'Européen un allié et un maître [qu']ils avaient suivi avec loyauté et fraternité». L'auteur compare implicitement la situation des harkis à celle des anciens combattants de Salò à la fin du second conflit mondial.

Nous, les Italiens, nous pouvons aisément imaginer ce qui s'est passé ensuite : le déchaînement des plus bas instincts de férocité bestiale et de cruauté sur les femmes et les enfants, coupables seulement d'avoir servi avec fidélité et honneur un drapeau jusqu'à l'extrême limite. [...]. Tout ce qu'un homme pouvait faire à un autre homme a été effectué sur les harkis, qui viennent ainsi allonger la liste, déjà trop longue, des victimes de la cruauté rougess.

Nazzareno Mollicone passe sous silence les exactions commises par les fascistes de Salò pour mieux mettre en avant les crimes communistes, dans le contexte de guerre civile qui marqua le Nord de l'Italie durant les derniers mois de guerre ${ }^{56}$. La barbarisation de l'adversaire et l'emploi de termes à connotation stigmatisante mettent en lumière la radicalité de l'affrontement politique et la charge émotive liée à l'évocation de ces conflits.

Les harkis ne sont pas les seuls à bénéficier de la sollicitude des Italiens. Parallèlement, Ordine Nuovo dénonce le traitement considéré comme indigne réservé par les autorités françaises aux anciens officiers de l'Algérie française et loue leur supposé sacrifice pour la cause. De nouveaux martyrs

53. Sur ce point, pour un développement plus détaillé, nous nous permettons de renvoyer à P. Picco, Histoire entrecroisée des extrêmes droites françaises et italiennes : cultures politiques, itinéraires, réseaux (1960-I984), thèse de doctorat (dir. O. Faron et M. Zancarini-Fournel), Université Paris-Sorbonne, 20I3, p. I9I et suivantes.

54. Nazzareno Mollicone est né à Pontecorvo en 1939. Il fut un militant actif du Centro studi Ordine Nuovo de Rome du début des années I960 à 1969, année où il rejoint le MSI avec la frange rautienne du mouvement.

55. N. Mollicone, «La tragedia degli harkis», art. cité, p. 49.

56. C. Pavone, Une guerre civile. Essai historique sur l'éthique de la Résistance italienne, Paris, Seuil, «Univers historique", 2005 (I99I); R. De Felice, Les noirs et les rouges : Mussolini, la République de Salò et la résistance 1943-1945, Genève, Georg, 1999. 
sont ainsi intégrés au sein du panthéon de l'extrême droite italienne. Clemente Graziani, dirigeant d'Ordine Nuovo, prend lui-même la plume, en avril 1963, pour exalter la lutte menée par l'organisation secrète et le souvenir du départ des Français d'Algérie, contraints selon lui par les «institutions internationales» à l'abandon de leurs terres ${ }^{57}$. Il exalte l'engagement des «officiers français, de Salan et Jouhaux, de Zeller à Gardy, de Broizat à Argoud, à Gardes, à Godard, à Denoix de Saint-Marc qui se sont sacrifiés dans la tentative désespérée de conserver pour la France et l'Europe les terres de l'Algérie française ${ }^{58}$ ». La mention de Zeller, Challe et Denoix de Saint-Marc, qui participèrent à l'organisation du putsch d'Alger, mais ne furent jamais membres de l'OAS, met en lumière un certain amalgame, voire une méconnaissance de la composition - complexe - de l'organisation. C'est certainement la "puissance mobilisatrice du mythe» qui est ici invoquée, sans qu'une connaissance précise des individus évoqués ne soit nécessaire ${ }^{59}$.

Parallèlement, le périodique exalte la figure de Bastien-Thiry, «condamné à mort par de Gaulle» après l'attentat du Petit-Clamart et «mort héroïquement». L'auteur se félicite qu'il

[...] a [it] refusé d'avoir les yeux bandés préférant regarder bien dans les yeux les hommes qui s’apprêtaient à le fusiller. [...] C'est le quatrième condamné de l'OAS qui meurt à l'aube, dans un fort militaire : Bastien-Thiry a suivi dans son sacrifice Piegts, Dovecar et le lieutenant Degueldre, dont le comportement fut si héroïque que le peloton d'exécution hésita longtemps avant d'ouvrir le feu ${ }^{60}$.

La dimension sacrificielle de ces exécutions transfigure la mort des activistes, et leur donne un sens supérieur, encore amplifié par l'héroïsation des condamnés.

Ordine Nuovo n'est pas le seul groupe à faire l'éloge des martyrs de l'OAS : les Formazioni nazionali giovanili tentent d'organiser le I8 mars I963 à Rome une messe à la mémoire de Bastien-Thiry, qualifié dans un tract d' "héroïque combattant pluri-décoré» dont la vie fut fauchée par le "plomb gaulliste». Ils se heurtent à l'opposition du vicariat de Rome mais passent outre. Conformément à un rituel fasciste, ils se réunissent autour de l'autel, scandent le nom du colonel Bastien-Thiry et répondent par

57. C. Graziani, «La guerra rivoluzionaria», Ordine Nuovo, vol. IX, no 2, avril 1963, p. II-27.

58. Ibid., p. 2I.

59. R. Girardet, Mythes et mythologies politiques, Paris, Seuil, I986, p. I3-I4.

60. Aryas, "Giorno per giorno", Ordine Nuovo, vol. IX, n 2, avril 1963, p. 62. Les hommes cités, membres de l'OAS, furent condamnés à mort par de Gaulle : Claude Piegts et Bobby Dovecar, anciens Delta, furent fusillés le 7 juin 1962. Roger Degueldre, ancien du i ${ }^{\text {er }}$ REP, dirigeant des commandos Delta, fut exécuté le 6 juillet 1962. Jean-Marie Bastien-Thiry fut fusillé le II mars 1963. 
l'injonction "présent» en faisant le salut fasciste ${ }^{61}$. Le "culte des morts au champ de bataille, des martyrs", qui "occupait une grande place dans [la] liturgie politique ${ }^{62}$ " fasciste est ainsi repris, à l'identique, ritualisé, et met en lumière la réappropriation du combat de l'OAS par les militants italiens. Cette mise en scène est doublée de la publication d'un tract, dans lequel les militants revendiquent «le droit à l'insurrection et à la lutte contre la tyrannie ${ }^{63}$ ", reprenant la rhétorique martyrologique développée par l'OAS. La ritualisation de l'injonction "présent», partagée par les Français ${ }^{64}$, contribue en outre à "contrecarrer l'ensevelissement d'un passé $^{65}$, tandis que la mort est, dans un même mouvement, exaltée et niée.

La dimension sacrificielle de ce combat et son héroïsation comportent en outre un rôle mobilisateur ${ }^{66}$. En 1964, deux ans après la fin du conflit algérien, le dirigeant du Centro studi Ordine Nuovo met en lumière la pérennité de ce mythe mobilisateur.

Les jeunes qui ne s'enthousiasment pas outre mesure à propos de la "polémique» sur la frontière entre l'Italie et l'Autriche, sont les mêmes qui en revanche se sont énormément enthousiasmés pour l'action de l'OAS en Algérie et en France. Et certains ont fait suivre les actes aux paroles, ils ont fourni armes et explosifs, ils ont aidé à l'expatriation des paras et des légionnaires condamnés à mort par les tribunaux gaulliens et des milliers de jeunes étaient prêts à en faire autant si seulement les liaisons avaient été meilleures et s'ils en avaient eu le temps. [...] Si les parachutistes étaient partis d'Alger et avaient occupé Paris, et si, en France, la guerre civile avait éclaté, cinquante mille volontaires au moins seraient partis combattre depuis l'Italie — à la faveur de cette révolution nationale contre le régime des partis de la IV République ${ }^{67}$.

S'il est probable, nous l'avons vu plus haut, que le Centro studi Ordine Nuovo ait aidé matériellement l'OAS, il apparaît en revanche parfaitement impossible qu'il ait été, à un quelconque moment de son existence, capable de mobiliser un nombre aussi important de militants.

6I. Casa della Memoria, Dossier de procédure pénale nº 9I/97 mod. 2I, ADCPP, fasc. «Formazioni nazionali giovanili », lettre «réservée» de la Préfecture de police de Rome au ministère de l'Intérieur, DGPS, div. AA. RR. et AA. GG., I8 mars 1963 .

62. G. L. Mosse, L'image de l'homme..., ouvr. cité, p. I83.

63. Casa della Memoria, Dossier de procédure pénale nº 9I/97 mod. 2I, ADCPP, fasc. «Formazioni nazionali giovanili», tract du FNG "Nuova Europa», I8 mars 1963.

64. La ritualisation de l'injonction "présent" dont les militants italiens affublent ceux qu'elle considère comme ses martyrs est partagée par les Français, en témoigne le rappel à la mémoire de Bastien-Thiry: "Hommage. Le II mars I963 à 6 h I2, le Colonel Bastien-Thiry a été fusillé par les soldats du régime. Il a payé de sa vie son combat. [...] Pour l'Algérie française, pour l'Europe de demain, pour la Révolution, BastienThiry, présent!», Europe-Action hebdomadaire, n 6I, lundi 15 mars 1965 , p. I.

65. P. Braud, L'émotion en politique, Paris, Presses de Sciences Po, 1996, p. 95.

66. R. Girardet, Mythes..., ouvr. cité, p. I3-I4.

67. P. Rauti, «L'Europa e il terzo mondo", Ordine Nuovo, vol. X, n 5-6, juin-juillet 1964, p. I-II. 
Le mythe OAS, doublé de celui des "paras» de la Légion étrangère, perdure et sa postérité s'étend jusque dans les années 1970 où il réapparait périodiquement dans les publications et tracts diffusés par les groupes d'extrême droite ${ }^{68}$. La culture du refus qui imprègne les mentalités de leurs militants et plus spécifiquement celle des franges extra-parlementaires explique en partie l'adhésion au mythe et l'écho qu'il trouve dans ses périodiques. La notion de résistance, qui se manifeste plus particulièrement à l'égard du «système», est ainsi au cœur de la construction politique et culturelle des individus qui gravitent au sein de l'extrême droite. Neuf ans après la fin de la guerre d'indépendance algérienne, l'OAS demeure une référence pour les groupes terroristes italiens ${ }^{69}$.

Le combat de l'OAS inaugure une série de réappropriations de mythes communs et la formation d'une mémoire militante similaire des deux côtés des Alpes. Le mythe du "para» se rattache à celui du guerrier dorique en son temps mobilisé par les SS d'Himmler ${ }^{70}$, du chevalier du Saint-Empire romain germanique, du légionnaire de la RSI, du SS ${ }^{71}$, et celui de l'OAS au combat d'une minorité avant-gardiste pour une cause perdue. Les activistes de l'organisation secrète participent de l'européanisation d'un patrimoine idéologique commun aux extrêmes droites, favorisé par la circulation de périodiques qui soutiennent directement ou indirectement la cause de l'OAS de part et d'autre des Alpes. Les circulations idéologiques et culturelles contribuent à former des éléments d'une culture politique commune entre Français et Italiens d'extrême droite.

68. V. P. Rauti, "Appunti per una tattica e una strategia degli anni '70», Ordine Nuovo, vol. I, n I (nouvelle série), 1970, p. 5-17; «Né onore né gloria», La Fenice, vol. II, n 5, 22 septembre 1972, p. 3; N. Mollicone, «Dieci anni fa : l'abbandono dell'Algeria», L'Italiano, vol. XIII, n I5, I972, p. 490-49I.

69. Casa della Memoria, Lettre de la Préfecture de Rome, bureau politique, au procureur de la République, tribunal civil et pénal, Rome, I ${ }^{\text {er }}$ juin I973, tract d'Avanguardia Nazionale, Trente, I5 octobre I97I.

70. J. Chapoutot, Le national-socialisme et l'Antiquité, Paris, PUF, 1998, p. 346.

7I. Caesar, "Romanità e germanesimo", Ordine Nuovo, vol. II, n 9, septembre 1956, p. I4-I5. 